Sprachkultur und Bürgertum 


\section{Angelika Linke}

\section{Sprachkultur und Bürgertum}

Zur Mentalitätsgeschichte des 19. Jahrhunderts 
Publiziert mit Unterstützung des Schweizerischen Nationalfonds zur Förderung der wissenschaftlichen Forschung

Die Deutsche Bibliothek - CIP-Einheitsaufnahme

Linke, Angelika:

Sprachkultur und Bürgertum : zur Mentalitätsgeschichte

des 19. Jahrhunderts / Angelika Linke.

- Stuttgart ; Weimar : Metzler, 1996

ISBN 978-3-476-01424-5

ISBN 978-3-476-01424-5

ISBN 978-3-476-03641-4 (eBook)

DOI 10.1007/978-3-476-03641-4

Dieses Werk einschließlich aller seiner Teile ist urheberrechtlich geschützt.

Jede Verwertung außerhalb der engen Grenzen des Urheberrechtsgesetzes ist ohne

Zustimmung des Verlages unzulässig und strafbar. Das gilt insbesondere für Vervielfältigungen, Übersetzungen, Mikroverfilmungen und die Einspeicherung und Verarbeitung in elektronischen Systemen.

(C) 1996 Springer-Verlag GmbH Deutschland

Ursprünglich erschienen bei J.B. Metzlersche Verlagsbuchhandlung

und Carl Ernst Poeschel Verlag GmbH in Stuttgart 1996 


\section{Vorwort}

Ich habe das vorliegende Buch gerne geschrieben. Dass dies so ist, hat nicht nur, aber auch - und vielleicht sogar zur Hauptsache - damit zu tun, dass ich bei meiner Arbeit immer wieder Anregung und Unterstützung erfahren habe, von vielen und von sehr verschiedenen Seiten.

In materieller Hinsicht massgeblich an diesem Buch beteiligt ist der Schweizerische Nationalfonds zur Förderung der wissenschaftlichen Forschung, der sein Entstehen durch ein grosszügiges Stipendium ermöglicht und auch die Drucklegung finanziell unterstützt hat.

Auf ihre Weise materiell beteiligt sind die Institutionen und Privatpersonen, die mir zum konkreten sprachhistorischen Gegenstand des Buches verholfen haben - zu den Quellentexten. Die aufwendige Suche danach wäre für mich wohl noch mühsamer geworden, hätte ich nicht eine Reihe von Institutionen von ihrer hilfreichen Seite oder richtiger: in diesen Institutionen hilfreiche Menschen kennengelernt. Für ihre freundliche Unterstützung danke ich Konrad Vanja und Roland Wohlfart vom Museum für Volkskunde, Berlin, Thomas Föhl vom Berlin Museum, Berlin, dem Landesarchiv Berlin, dem Werkbundarchiv Berlin und der Staatsbibliothek Berlin. Walter Kempowski danke ich für die Gastfreundschaft in seinem Privat-Archiv in Nartum, welches für mich eine Fundgrube war, und Dorothee Gerhardt dafür, dass sie mich in Gamburg auf den Dachboden geführt hat.

Was die inhaltliche Seite des Buches anbelangt, so hat es - am Schnittpunkt von Sprachgeschichte und Sozialgeschichte angesiedelt - nur entstehen können, weil ich in beiden Wissenschaftsbereichen sowohl fachliche als auch persönliche Unterstützung und Förderung erfahren habe. Mein Dank gilt hier in erster Linie Rudolf Braun, bei dem ich sozialgeschichtliches Denken gelernt habe - auch in Gesprächen, die nichts mit Sozialgeschichte zu tun hatten. Ebenso Dank schulde ich Dieter Cherubim und Klaus J. Mattheier, die mein Interesse an der Sprachgeschichte des 19. Jahrhunderts kontinuierlich unterstützt und mir Gelegenheit zum fachlichen Austausch im kleineren wie im grösseren Kreis geboten haben.

Was mich selbst anbelangt, so habe ich während meiner Arbeit - und vor allem in den offenbar unausweichlichen Härtephasen - viel Rückenstützung und Seelenpflege von 'meinen Uni-Frauen`, vor allem von Silvia Derrer und Katrin Wiederkehr-Benz erfahren; ihnen möchte ich an dieser Stelle herzlich danken. Felicitas Spuhler danke ich für ihre Korrekturarbeit und für alle anderen Selbst- 
verständlichkeiten, Willibald Steinmetz und Dorothee Kohler-Luginbühl für Anerkennung im richtigen Moment und meinen Kolleginnen und Kollegen vom Deutschen Seminar der Universität Zürich (allen voran Markus Nussbaumer) dafür, dass sie den linguistischen Alltag so äusserst annehmlich machen.

Dass es aber überhaupt so weit gekommen ist, verdanke ich meinen linguistischen Lehrern in Zürich: Harald Burger, ohne den ich wohl gar nicht zur Linguistik gekommen, und Horst Sitta, ohne den ich vielleicht nicht dabei geblieben wäre.

Was ich Horst Sitta insgesamt schuldig bin - linguistisch, institutionell und in vieler Hinsicht darüber hinaus - lässt sich nicht so einfach sagen. Aber ich danke es ihm von Herzen.

Allerdings: Was von vielen wissenschaftlichen Büchern behauptet wird, gilt auch für dieses: Es wäre ohne eine bestimmte Person und ohne die Inspiration einer bestimmten - durchaus unwissenschaftlichen - Umgebung vielleicht gar nicht, zumindest aber nicht so, zustandegekommen. In meinem Fall handelt es sich dabei um eine Tante mit einem Dach in Berlin. Ihr ist dieses Buch gewidmet. 


\section{Inhalt}

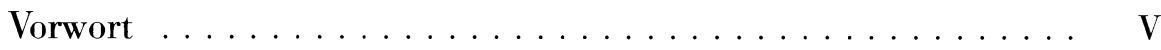

Einleitung $\ldots \ldots \ldots \ldots \ldots \ldots \ldots \ldots \ldots \ldots \ldots \ldots \ldots \ldots \ldots \ldots$

\section{Teil I: Vorklärungen}

1. Linguistische Aspekte . . . . . . . . . . . . . . 9

1.1 Sprache als Sozialsymbol . . . . . . . . . . . . . . . 11

1.2 Zu den Berührungspunkten von Pragmatik, Soziolinguistik und Sprachgeschichte . . . . . . . . . . . . . . . . 13

1.3 Umrisse einer pragmatisch orientierten Sprachgeschichts-

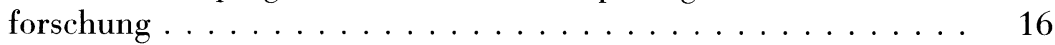

2. Historische Aspekte . . . . . . . . . . . . . . . . . 19

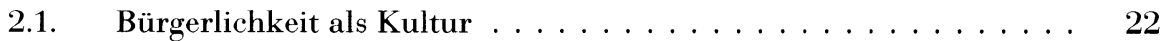

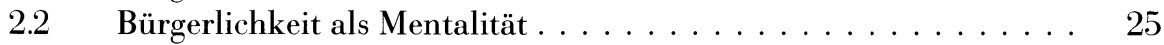

2.3 Bildungsbürgertum als Orientierungsformation $\ldots \ldots \ldots \ldots 27$

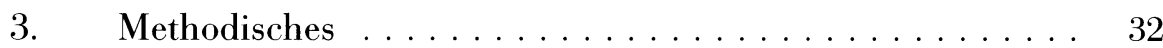

3.1 Historische Pragmatik und die Analyse kollektiver Dispositionen . 32

3.2 Quellen ......................... 34

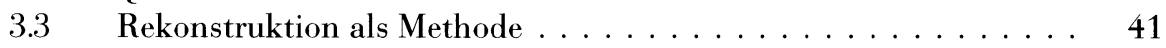

4. Voraussetzungen und Bedingungen einer bürgerlichen Sprachkultur im 19. Jahrhundert . . . . . . . . . . . 46

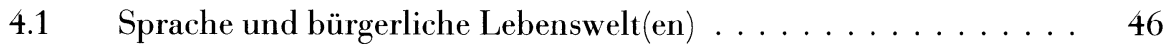

4.1 .1 Der »vernünftige« Diskurs . . . . . . . . . . . . . . . . 49

4.1.2 Die »Mannichfaltichkeit» der Verkehrskreise . . . . . . . . . . 50

4.1.3 Veränderung familiärer Kommunikationskonstellationen . . . . . 52

4.1.4 Sprache und Bildungsreligion $" \ldots \ldots \ldots \ldots \ldots \ldots \ldots_{54}$

4.2 Sprache als Medium bürgerlicher Repräsentationskultur . . . . . 56 


\section{Teil II: Analysen}

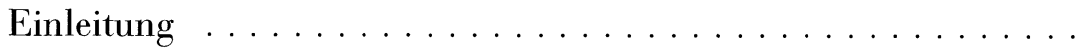

5. Leiblichkeit als Standeskultur: Körperbarrieren zwischen Adelsgesellschaft und Bürgertum . . . . . . . . . . 63

$5.1 \quad$ Bewegungskultur und adlige Standesrepräsentation . . . . . . 65

5.2 Die Exerzitien als Basis gentiler »Körperkunst" . . . . . . . . 67

5.2.1 Das Reiten »nett nach der Mensur und Tact . . . . . . . . . . . 67

5.2.2 Das »zierliche« Fechten . . . . . . . . . . . . . . . . . . 67

5.2 .3 Das violente« Voltigieren . . . . . . . . . . . . . . . . 68

5.2 .4 Das repräsentative Ballhausspiel . . . . . . . . . . . . . . . 69

$5.3 \quad$ Konfigurationen der Adelsgesellschaft $\ldots \ldots \ldots \ldots \ldots \ldots$

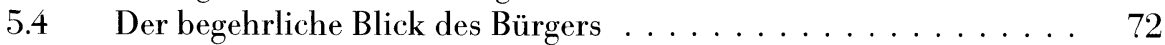

$5.5 \quad$ Entwürfe einer bürgerlichen Bewegungskultur . . . . . . . 77

5.5.1 »Natur« contra »Afectation« . . . . . . . . . . . . . . . . . 77

5.5.2 Dimensionen bürgerlicher Körpergestik . . . . . . . . . . . . 80

5.6 Bewegungskultur und Mentalitätsgeschichte $\ldots \ldots \ldots \ldots . \ldots 8$

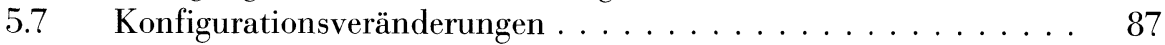

5.8 Vom tanzenden Höfling zur tanzenden Bürgerin: Die bürgerliche Anverwandlung eines Bewegungszeremoniells _. . . . . . . . 90

5.8.1 Der Tanz als adliges Exerzitium und als »Hof=Kunst $\ldots \ldots \ldots . .90$

5.8.2 Der Walzer als »bürgerlicher« Tanz? . . . . . . . . . . . . . . . 95

5.9 Redimensionierung, Abwertung und Umwertung des Körperlichen im bürgerlichen Kontext . . . . . . . . . . . . . . . . . 98

5.9.1 Die Delegation des Körperzeremoniells an die Bürgerin _ . . . . 98

5.9 .2 Körpergestik im Dienst der Geschlechterinszenierung . . . . . 100

6. Vom Compliment zum Gruss: ein Exempel . . . . . . . . . . 104

6.1 Das Compliment als Höflichkeit »in Worten und Geberden« . . . . . 104

6.2 Redimensionierungen . . . . . . . . . . . . . . . . . . . 108

6.2.1 Redimensionierung des »Wort-Compliments" . . . . . . . . . . . 109

6.2.2 Redimensionierung des "Geberden-Compliments" . . . . . . . . 113

6.3 Vom Compliment zum Gruss: Entwicklungen im 19. Jahrhundert . 120

6.4 Die»Versprachlichung« des Compliments zur verbalen Geste . . . 129

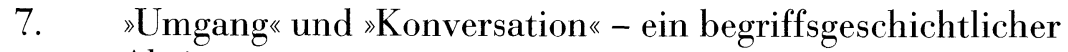
Abriss . . . . . . . . . . . . . . . . . . . . . . 132

7.1 »Ob man in Conversation reden soll $\ldots \ldots \ldots \ldots \ldots$. . . . . . . 132

7.2 »Conversation « im 19. Jahrhundert . . . . . . . . . . . . . . . . 135

7.3 „Conversationsstücke« . . . . . . . . . . . . . . . 138

7.4 $\mathrm{Zu}$ den ethisch-ästhetischen und sozial-ständischen Konnotationen von `Konversation` . . . . . . . . . . . . . . . . . . . . . 142 
7.5 Die Assoziation von 'Konversation` und 'Bildung` im 19. Jahrhundert . . . . . . . . . . . . . . . . . . . . . . . 144

7.6 Vom gesellschaftlichen Umgang zum gesellschaftlichen Gespräch . 149

8. Der Verlust der schönen Stimme . . . . . . . . . . . . . 151

8.1 Die ästhetisch-sinnliche Dimension der Stimme . . . . . . . . . . 152

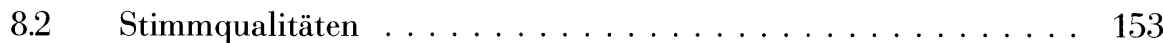

8.3 Die normative Dimension der Aussprache . . . . . . . . . . 156

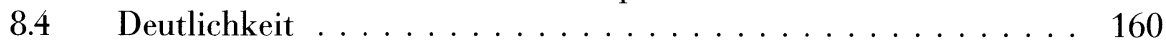

8.5 Lautstärke . . . . . . . . . . . . . . . . . . 161

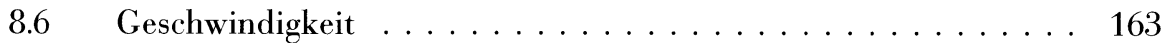

8.7 Wahrnehmungswandel und Normenwandel . . . . . . . . . . 164

9. Gesellschaft, Gespräch und bürgerliche Geselligkeit . . . . 170

9.1 Das Gesellschaftszimmer . . . . . . . . . . . . . . . 170

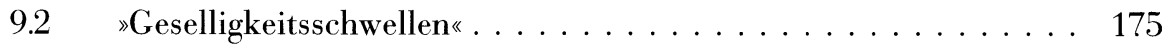

9.3 Die Visite als Gesellschaftsritual . . . . . . . . . . . . . . 178

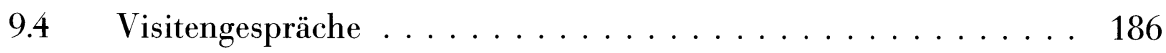

9.5 Das »Gespräch in Gesellschaft $\ldots \ldots \ldots$. . . . . . . . . . . . . . . 191

9.5.1 Das Gespräch als Medium bürgerlicher Vergesellschaftung . . . . 191

9.5 .2 Die Gesprächsthemen . . . . . . . . . . . . . . . . . . 196

9.5 .3 Verbotene Themen ....................... 203

9.5 .4 Das Gespräch als kollektive Leistung . . . . . . . . . . . . . . . . 207

9.6 Die Rolle der Frauen im gesellschaftlichen Gespräch . . . . . . . . 212

9.6.1 Sittenwächterin und Anstandslehrerin . . . . . . . . . . . . . . 212

9.6 .2 Die Gesprächsarbeiterin . . . . . . . . . . . . . . . 214

9.6 .3 Die Garantin für Gesprächsdisziplin . . . . . . . . . . . . . 217

9.7 Das Gespräch als Gesellschaftsspiel . . . . . . . . . . . . . . 220

9.8 Bürgerliche Gesprächsspiele . . . . . . . . . . . . . . . . 224

9.8.1 Der unbürgerliche Versuch einer Leichtigkeit des Seins . . . . . . 224

9.8.2 Distinktion, Integration und herstellbare Harmonie . . . . . . . . 227

10. Die Liebe im Dativ . . . . . . . . . . . . . . . . . 231

10.1 Sprachliche Bildung als Bürgerpflicht . . . . . . . . . . . . 234

10.2 Zur sozialen Hierarchisierung von Dialekt und Standardsprache . 238

10.3 Dialekt und bürgerliche Sprachsozialisation . . . . . . . . . . . 241

10.4 Schreckgespenst und Hätschelkind bürgerlicher Sprachaufmerksamkeit . . . . . . . . . . . . . . . . . . . . . . . 244

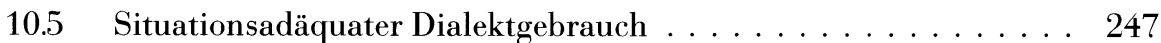

10.6 Gesellschaftliche Unterhaltung . . . . . . . . . . . . . . . . . 249

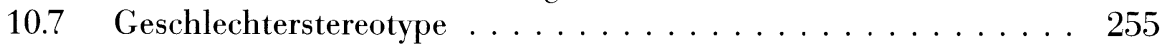

10.8 Zur Symbolfunktion des Dialekts . . . . . . . . . . . . . . 260 
11. Zur sprachlichen Kodierung bürgerlichen Lebensgefühls . . 265

11.1 Zur sprachlichen Kodierung von Gefühlen . . . . . . . . . . . 265

11.2 Tagebuchtexte: Privatheit, soziale Rolle und die »Traditionen des Sprechens" . . . . . . . . . . . . . . . . . . . 267

11.3 Ein bürgerliches Gesellschaftswort . . . . . . . . . . . . 270

11.4 Die sprachliche Aneignung und Entfaltung bürgerlichen Lebens-

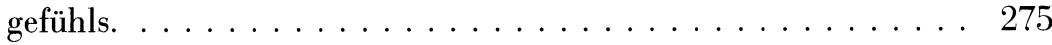

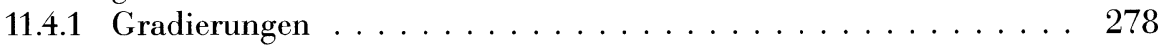

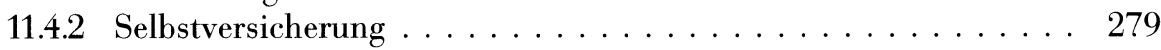

11.4 .3 peer-group-Funktion . . . . . . . . . . . . . . . . . . 281

11.4.4 Das Amüsement als soziale Leistung - das richtige< Reden darüber als soziale Kompetenz . . . . . . . . . . . . . . . . 282

11.5 Phraseologisierung als Festschreibung kultureller Konzepte? . . . . 285

11.6 Sozialsemiotische Dimensionen . . . . . . . . . . . . . . 286

11.7 Sozialgeschichtliche Dimensionen: die Frage nach der »Bürgerlichkeit» . . . . . . . . . . . . . . . . . 288

12. Sprachkultur und bürgerliche Kinderstube . . . . . . . 291

12.1 Vom täglichen Schreiben . . . . . . . . . . . . . . . . . 291

12.2 Schreibrituale . . . . . . . . . . . . . . . . . . . 294

12.3 Sprachliche Leistung als Geschenk der Kinder an die Eltern . . . . 299

12.4 Briefe im Dienst der Beziehungspflege . . . . . . . . . . . . . 303

12.5 Die emotionale Besetzung von Texten und Schreibprodukten . . . . 309

12.6 Sprachliche Kindheitsmuster . . . . . . . . . . . . . . 313

Schluss . . . . . . . . . . . . . . . . . . . . . 317

Bibliographie $\ldots \ldots \ldots \ldots \ldots \ldots \ldots \ldots \ldots \ldots \ldots \ldots \ldots$

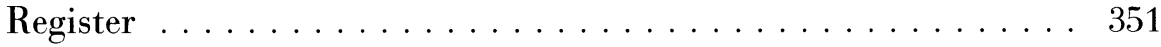

